\title{
Signal Timing Optimization Based on Fuzzy Compromise Programming for Isolated Signalized Intersection
}

\author{
Dexin Yu, ${ }^{1,2,3}$ Xiujuan Tian, ${ }^{2}$ Xue Xing, ${ }^{2}$ and Shutao Gao ${ }^{2}$ \\ ${ }^{1}$ State Key Laboratory of Automobile Dynamic Simulation, Jilin University, Changchun 130022, China \\ ${ }^{2}$ College of Transportation, Jilin University, Changchun 130022, China \\ ${ }^{3}$ Jilin Province Key Laboratory of Road Traffic, College of Transportation, Jilin University, Changchun 130022, China
}

Correspondence should be addressed to Dexin Yu; yudx@jlu.edu.cn

Received 8 December 2015; Revised 3 February 2016; Accepted 29 February 2016

Academic Editor: Konstantinos Karamanos

Copyright (C) 2016 Dexin Yu et al. This is an open access article distributed under the Creative Commons Attribution License, which permits unrestricted use, distribution, and reproduction in any medium, provided the original work is properly cited.

\begin{abstract}
In order to optimize the signal timing for isolated intersection, a new method based on fuzzy programming approach is proposed in this paper. Considering the whole operation efficiency of the intersection comprehensively, traffic capacity, vehicle cycle delay, cycle stops, and exhaust emission are chosen as optimization goals to establish a multiobjective function first. Then fuzzy compromise programming approach is employed to give different weight coefficients to various optimization objectives for different traffic flow ratios states. And then the multiobjective function is converted to a single objective function. By using genetic algorithm, the optimized signal cycle and effective green time can be obtained. Finally, the performance of the traditional method and new method proposed in this paper is compared and analyzed through VISSIM software. It can be concluded that the signal timing optimized in this paper can effectively reduce vehicle delays and stops, which can improve traffic capacity of the intersection as well.
\end{abstract}

\section{Introduction}

Due to increase of vehicle numbers and growing traffic demand, traffic problems are becoming more and more serious. It is well known that the implementation of traffic signal control at intersections is one of the effective methods to relieve the series of traffic problems [1]. Therefore, researching the signal timing optimization methods is of great significance to improve the operation efficiency of the intersections.

Signal timing optimization has been highly concentrated in many years and there have been many researches in this field. When optimizing the signal timing parameters, it is needed to consider various performance indexes comprehensively, such as road capacity, vehicle delays, vehicle stops, and queue length. Early researchers were mostly focused on the optimization of a single index, typically represented by traditional Webster signal timing method of which the optimization objective was to minimize average vehicle delays [1]. For isolated signalized intersections, Ceder and Reshetnik [2] proposed an algorithm to minimize vehicle queues, which was proved efficient. Wang and Yang [3] established a signal timing optimization model to minimize average delays and applied genetic algorithm to search for optimal signal timing parameters. The results showed that the proposed method could obtain lower average delay and stops than using Synchro software. Under oversaturated state, Liu et al. [4] built a dynamic linear programming optimization model with the objective of maximizing traffic capacity at the intersection. Contributing to the deepening of the sustainable development concept, the optimization objective of signal timing control was changed from single objective to multiple objectives gradually. Based on data fusion, Deng et al. [5] proposed a new multiobjective optimization control strategy for urban signalized intersections. Vehicle delay, queue length, and data fusion technology were considered in the strategy, which was proved to be consistent with the traffic flow characteristics in China. Focused on isolated intersection, Jiao and Sun [6] presented a multiobjective signal control model framework based on dynamic O-D estimation. A state-space model was established first. And then dynamic turning movements were estimated. Compared with Webster 
method, the results were close to actual values. Anderson et al. [7] specially researched the optimization objectives of signal control system systematically, which indicated that signal control strategies should be flexible to adapt to various traffic states. The importance of each objective was supposed to adjust according to diverse traffic states. Schmöcker et al. [8] chose three evaluation indexes as optimization objectives, including average vehicle delays, average queue length, and pedestrian waiting timing. And then a multiobjective optimization function was established. Bellman-Zadeh fuzzy logic was employed to convert the model to single objective function which was finally solved by genetic algorithm. Li et al. [9] optimized the signal timing scheme of oversaturated intersections and the optimization objectives were maximum throughput vehicle numbers and minimum vehicle queue rate. Employing fuzzy logic approach, Murat and Gedizlioglu [10] proposed a multiphased signal control optimization method of isolated intersections.

In summary, the existing researches did not comprehensively consider enough factors. This may lead to the fact that one or two indexes were optimal but others were not optimal. The signal timing for isolated intersections in most current researches was optimized by adding several performance indexes together simply and giving diverse weight values to different indexes subjectively. It was not appropriate because the indexes were often on different dimensions. In addition, the weight of each index could not be adjusted according to traffic flow state. Therefore, it is essential to optimize multiple objectives at the same time and choose a more eclectic scheme to make each objective in a reasonable range.

To overcome the drawbacks mentioned above, a new signal timing optimization method for isolated signalized intersections is proposed in this paper based on fuzzy programming. The overall benefits of the intersection are considered in this paper. For establishing a multiobjective function to optimize signal timing parameters, several performance indexes are chosen first, including traffic capacity, total vehicle delays, and vehicle stops in a cycle, as well as exhaust emissions. In order to avoid the subjective factors as much as possible, fuzzy compromise programming is employed to give each optimization objective various weight coefficients. In this paper, weight coefficients can vary with different traffic flow states. When traffic flow is high, the main optimization objectives are to extremely increase the traffic capacity and reduce cycle vehicle delays. On the contrary, when traffic flow is low, the main optimization objectives are to reduce cycle vehicle delays and cycle stops. Then the multiobjective optimization problem is converted to a single objective optimization problem. To obtain the optimized signal cycle and effective green times, genetic algorithm is used to solve the model by MATLAB software. Finally, the signal timing scheme proposed in this paper is compared with other methods, employing the VISSIM simulation software. The results illustrate that the scheme proposed in this paper can effectively reduce vehicle delay and stops, improving the traffic capacity and the whole operation efficiency of the intersection.

The rest of this paper is organized as follows. Section 2 formulates the multiobjective programming model including optimization objectives selection presented in Section 2.1 and multiobjective programming modeling presented in Section 2.2. In Section 3, optimization objective functions are solved by fuzzy compromise programming and genetic algorithm. In Section 4, the model proposed in this paper is verified by choosing a typical intersection in Changchun, China. The results are compared with other traditional methods. Section 5 reveals the conclusions and recommendations for future research.

\section{Multi-Objective Programming Model}

2.1. Optimization Objectives Selection. It is known that the optimization goals of signal timing are to improve the passing efficiency and reduce vehicle delays and stops at the intersection. Therefore, considering the overall efficiency of the intersection, four traffic performance indexes including traffic capacity, cycle vehicle delay, cycle vehicle stops, and exhaust emission are chosen as the optimization objectives to obtain the optimized signal timing in this paper.

Several assumptions are given to formulate the models. Firstly, the unsaturated intersections are focused on in this paper. The condition when the intersections are oversaturated is not included. Then assume that the distance between the adjacent intersections is long enough and the vehicle arrival of downstream intersection is little affected by the signal setting at the upstream intersection. At the analysis interval, vehicle arrival rate remains stable and obeys a certain traffic arrival distribution, usually Poisson distribution. Although vehicles queue may be formed due to the random arrival in some cycles, the arrival of vehicles keeps balance with vehicle dissipation in the whole analysis period.

2.1.1. Traffic Capacity of the Intersection. Based on existing researches, the intersection capacity can be calculated by adding the capacity of each approach, respectively [11]. Assume that an intersection contains $n$ key phases, and then the intersection capacity can be calculated by

$$
\text { Cap }=\sum_{i=1}^{n} S_{i} \times \lambda_{i},
$$

where Cap denotes the intersection capacity, $S_{i}$ is the saturation flow for a particular phase $i, n$ is the number of key phases at the intersection, and $\lambda_{i}$ is the proportion of effective green time for phase $i$ which is equal to the ratio of effective green time and cycle by

$$
\lambda_{i}=\frac{g_{e i}}{C}
$$

where $C$ denotes the cycle time of the signalized intersection, $g_{e i}$ is the effective green time for phase $i$, and others are the same as above. Then the whole intersection capacity can be obtained as follows:

$$
\text { Cap }=\sum_{i=1}^{n} \frac{S_{i} g_{e i}}{C} .
$$


2.1.2. Cycle Vehicle Delays. In urban road network, most of the vehicle delays are generated at signalized intersections. As a consequence, vehicle delays can reflect the state of the road network to some extent and it is often chosen as signal timing optimization objective. When calculating the vehicle delays at a signalized intersection, Webster model [1] is usually used of which the characteristics are that the model is only suitable for low saturation of traffic flow and assume that the vehicle accumulation is continuous. Therefore, vehicle average delay model [12] is selected as

$$
d_{i}=\frac{C\left(1-\lambda_{i}\right)^{2}}{2\left(1-y_{i}\right)}+\frac{1-\lambda_{i}}{2 q_{i}}+\frac{q_{i}}{2 S_{i} \lambda_{i}\left(S_{i} \lambda_{i}-q_{i}\right)},
$$

where $q_{i}$ denotes the traffic flow and $y_{i}$ denotes the flow ratio of phase $i$, respectively. Substitute formula (2) into formula (4) and the following formula can be obtained. The others are the same as above. Consider

$$
\begin{aligned}
d_{i}= & \frac{C\left(1-g_{e i} / C\right)^{2}}{2\left(1-y_{i}\right)}+\frac{1-g_{e i} / C}{2 q_{i}} \\
& +\frac{q_{i} C^{2}}{2 S_{i} g_{e i}\left(S_{i} g_{e i}-q_{i} C\right)} .
\end{aligned}
$$

Then the cycle total vehicle delay of the intersection can be calculated as follows:

$$
\begin{aligned}
D & =\sum_{i=1}^{n} C q_{i} d_{i}=\sum_{i=1}^{n} C\left[\frac{q_{i} C\left(1-g_{e i} / C\right)^{2}}{7200\left(1-y_{i}\right)}+\frac{1-g_{e i} / C}{7200}\right. \\
& \left.+\frac{q_{i}^{2} C^{2}}{7200 S_{i} g_{e i}\left(S_{i} g_{e i}-q_{i} C\right)}\right] .
\end{aligned}
$$

2.1.3. Cycle Vehicle Stops. Due to the influence of signal light, vehicles often need to stop when approaching the intersection. So vehicle stops are also an important index to measure the traffic state of the intersection. The steady state model [13] proposed by Akçelik and Rouphail is employed to calculate vehicle stops as follows:

$$
\begin{aligned}
h_{i} & =0.9\left(\frac{1-\lambda_{i}}{1-y_{i}}+\frac{e^{k}}{2 q_{i} C\left(1-x_{i}\right)}\right), \\
k & =\frac{-1.33\left(1-x_{i} \sqrt{S_{i} q_{i}}\right)}{x_{i}},
\end{aligned}
$$

where $h_{i}$ and $x_{i}$ denote the vehicle stop rate and saturation level of phase $i$, respectively. Then the total vehicle stops in a cycle $M_{\text {stop }}$ can be obtained as follows:

$$
\begin{aligned}
M_{\text {stop }} & =\sum_{i=1}^{n} h_{i} q_{i} C \\
& =\sum_{i=1}^{n} \frac{0.9 q_{i} C}{3600}\left(\frac{1-\lambda_{i}}{1-y_{i}}+\frac{e^{-1.33\left(1-x_{i} \sqrt{S_{i} q_{i}}\right) / x_{i}}}{2\left(1-x_{i}\right) q_{i} C}\right) .
\end{aligned}
$$

2.1.4. Vehicle Exhaust Emission. The exhaust pollutants generated in the process of driving have a great impact on environment. For this reason, vehicle exhaust emission is taken as the forth optimization objective. The vehicle exhaust emissions at the intersection consist of two parts. One part is the idle exhaust emissions generated on the import approaches and another part is the exhaust emissions discharged in the intersection area $[14,15]$. Hence, vehicle exhaust emission can be formulated as follows:

$$
\begin{aligned}
E_{j}= & \frac{1}{3600} \sum_{i=1}^{n}\left(\mathrm{EFI}_{i}^{\mathrm{pcu}} \times q_{i} \times d_{i}\right) \\
& +\sum_{i=1}^{n}\left(\mathrm{EF}_{i}^{\mathrm{pcu}} \times q_{i} \times L_{i}\right),
\end{aligned}
$$

where $E_{j}$ denotes the emission of pollutant $j(\mathrm{~g} / \mathrm{h}), \mathrm{EFI}_{i}^{\mathrm{pcu}}$ is the pollutant emission factor for standard vehicle under idle condition $(\mathrm{g} /(\mathrm{pcu} \cdot \mathrm{h})), \mathrm{EF}_{i}^{\mathrm{pcu}}$ is the pollutant emission factor for standard vehicle $(\mathrm{g} /(\mathrm{pcu} \cdot \mathrm{km})), d_{i}$ is the average vehicle delay time for phase $i(\mathrm{~s} / \mathrm{pcu})$, and $L_{i}$ is the length of the approach for phase $i(\mathrm{~km})$. Usually, the values of $\mathrm{EFI}_{i}^{\mathrm{pcu}}$ and $\mathrm{EF}_{i}^{\mathrm{pcu}}$ are $5 \mathrm{~g} /(\mathrm{pcu} \cdot \mathrm{h})$ and $45 \mathrm{~g} /(\mathrm{pcu} \cdot \mathrm{km})$, respectively. Substitute the average delay into formula (10), and the final emission model could be obtained.

2.2. Multiobjective Programming Modeling. Considering the whole operation efficiency of the intersection, four objective functions are established of which the objectives are maximizing intersection traffic capacity, minimizing vehicle cycle delay, minimizing cycle stops, and minimizing exhaust emissions, denoted as $Z_{1}, Z_{2}, Z_{3}$, and $Z_{4}$, respectively.

The basic input parameters of the models are the saturation flow $S_{i}$, traffic flow $q_{i}$, the flow ratio $y_{i}$, and the length of the approach $L_{i}$ for phase $i(\mathrm{~km})$. And the output variables of the models are cycle time $C$ of the signalized intersection and the effective green time $g_{e i}$ for phase $i$. Consider

$$
\begin{aligned}
& Z_{1}=\min (- \text { Cap }), \\
& Z_{2}=\min D, \\
& Z_{3}=\min M_{\text {stop }} \\
& Z_{4}=\min E .
\end{aligned}
$$

Some constraints of the objective functions are formulated as follows which consider the actual traffic environment:

$$
\begin{array}{ll}
\text { s.t } & C_{\min } \leq C \leq C_{\max } ; \\
& g_{\min } \leq g_{e i} \leq g_{\max }, \quad 1 \leq i \leq n \\
& x_{\min } \leq \frac{y_{i}}{g_{e i}} C \leq x_{\max }, \quad 1 \leq i \leq n \\
& \sum_{i=1}^{n}\left(g_{e i}+l_{i}\right)=C, \quad 1 \leq i \leq n
\end{array}
$$

where the above constrains limit signal cycle length, effective green time, and saturation ratio of the intersection, respectively. The first constrain limits the signal cycle size in which 
$C_{\min }=15 n$ and $C_{\max } \leq 200$, where $n$ denotes the number of key phase sequences. The second constrain limits the effective green time size where $g_{\min }$ and $g_{\max }$ denote the minimum and maximum effective green time, respectively. The values of them can be determined according to the actual traffic environment [12]. The saturation size which is equal to the ratio of traffic flow and traffic capacity is limited by the third constraint. $x_{\min }$ and $x_{\max }$ denote the minimum saturation size and the maximum saturation size separately of which the values are often equal to 0.7 and 0.95 in order to avoid the waste of green time and too short green time [12].

\section{Optimization Objective Functions Solving}

Because solving multiobjective optimization problem is complex, so the combination method is often employed to solve this series of problems. By giving each optimization objective different weight coefficients, the multiobjective optimization problem can be transformed into single objective optimization problem. The dimensions of each objective are often different. Therefore, it is not reasonable to add multiple optimization objectives together simply. Then fuzzy compromise programming approach is brought in this paper.

3.1. Fuzzy Compromise Programming. Fuzzy compromise programming approach takes various objectives into consideration synthetically by marginally evaluating individual objectives and then globally evaluating all objectives. In the global subjective evaluation, the decisionmaker's preferences are reflected and various objectives are taken into account [16]. The multiobjective signal timing optimization function is converted to single objective function. According to different traffic state, the optimized signal cycle length and effective green time can be obtained.

The basic idea of fuzzy compromise programming method is to achieve overall optimum by calculating the distance between the feasible solution set and the ideal solution set and determining the feasible solution which is closest to the ideal solution as the final solution [17]. The objective functions in different dimensions can be changed into a dimensionless function through conversion. The values are limited between 0 and 1 . Different weight coefficients are given to different optimization objectives. The solving steps are as follows [18].

Step 1. Convert the objective functions to minimum forms under the limits of constraints. Calculate the minimum values and maximum values, respectively, first. For each particular objective $Z_{i}$ in the multiobjective programming problem $(i=$ $1,2, \ldots, k)$, the upper and lower bounds values are assigned which are denoted by $Z_{i \max }$ and $Z_{i \text { min }}$. In fact, for objective $Z_{i}, Z_{i \max }$ and $Z_{i \text { min }}$ represent the highest acceptable level and the aspired level of the achievement, respectively. Then the ideal value vector is constituted by the lower bounds values of all objective functions, denoted as $Z_{\min }$. Similarly, the opposite ideal value vector is constituted by the upper bounds values of objective functions, denoted as $Z_{\max }$ :

$$
\begin{aligned}
Z_{\min } & =\left(z_{1}, z_{2}, z_{3}, z_{4}\right) \\
& =\left(Z_{1 \min }, Z_{2 \min }, Z_{3 \min }, Z_{4 \min }\right), \\
Z_{\max } & =\left(z_{1}^{\prime}, z_{2}^{\prime}, z_{3}^{\prime}, z_{4}^{\prime}\right) \\
& =\left(Z_{1 \max }, Z_{2 \max }, Z_{3 \max }, Z_{4 \max }\right) .
\end{aligned}
$$

Step 2. Calculate the membership function of each optimization objective function. As it is known, there are several membership functions including linear, hyperbolic, and piecewise-linear [16]. For practicability and simplicity, the linear membership function [18] is adopted in this paper. With the upper and lower bounds values, marginal evaluation can be obtained for each objective $Z_{i}$. A mapping $u_{z i}(x)$ illustrates what degree the decision makes $i$ th objective close to the aspired solution, which is used to denote the membership function of objective $i$. The values of $u_{z i}(x)$ are between 0 and 1 , representing the degree of compatibility between the solved value and the aspired value. The marginal evaluation mapping can be obtained by the following formula:

$$
u_{z i}(x)= \begin{cases}1 & Z_{i}(x) \leq Z_{i \min } \\ \frac{Z_{i}(x)-Z_{i \max }}{Z_{i \min }-Z_{i \max }} & Z_{i \min }<Z_{i}(x)<Z_{i \max } \\ 0 & Z_{i}(x) \geq Z_{i \max } .\end{cases}
$$

Step 3. Calculate the weight coefficient of objective $i$ which is denoted as $\lambda_{i}$. The membership functions which are more important are constituted as aroup $F=\left\{u_{z 1}, u_{z 2}, \ldots, u_{z m}\right\}$. Equivalence class $\left\{C_{i} \mid 1 \leq i \leq n\right\}$ is built by using the relationship $\approx$. Choose one element from each equivalence class to form a collection which is denoted as $T=\left\{c_{1}, c_{2}, \ldots, c_{n}\right\}$. The values of the relationship function are determined by the evaluation function $v$ :

$$
\begin{aligned}
& v\left(c_{i}\right)=\alpha, \\
& v\left(c_{j}\right)=\beta, \\
& \quad\left(c_{i} \prec c_{j}\right), \\
& v\left(c_{i}\right)=v, \\
& v\left(c_{j}\right)=\gamma, \\
& v\left(c_{i}\right)=\delta, \\
& v\left(c_{j}\right)=\varepsilon, \quad\left(c_{i} \approx c_{j}\right),
\end{aligned}
$$

where the coefficients $\alpha, \beta, \nu, \gamma, \delta$, and $\varepsilon$ are all real numbers between 0 and 1 and the formulas $\delta<\alpha<\gamma<\beta<\varepsilon$ and $\alpha+\beta=\nu+\gamma=\delta+\varepsilon=1$ exist. 
Step 4. Define matrix $R_{a}$ according to the evaluation function $v$. And establish a relation matrix and denote it as $R$ :

$$
\begin{aligned}
& R_{a}(i, j)=0, \\
& R_{a}(j, i)=1, \\
& R_{a}(i, j)=1, \\
& R_{a}(j, i)=1, \\
& R_{a}(i, j)=0, \quad\left(c_{i} \approx c_{j}\right), \\
& R_{a}(j, i)=2, \\
& R(i, j)=\alpha, \quad\left(c_{i} \ll c_{j}\right), \\
& R(j, i)=\beta, \\
& R(i, j)=\delta, \\
& R(j, i)=\varepsilon, \\
& R(i, j)=v,
\end{aligned}
$$

Step 5. Then the weight coefficient $\lambda_{c_{j}}$ in which $c_{j}$ belongs to $T$ can be obtained as follows:

$$
\lambda_{c_{j}}=\frac{S_{L}\left(c_{j}, R\right)}{\sum_{c_{j} \in T} S_{L}\left(c_{j}, R\right)},
$$

where $S_{L}\left(c_{j}, R\right)$ denotes the boundary value of matrix $R$.

Step 6. Convert the multiobjective function to a single objective function:

$$
Z=\max \left[\sum_{i=1}^{m}\left(\lambda_{i} u_{z i}\right)^{p}\right]^{1 / p},
$$

where $p$ denotes the distance index of which the value can be different. According to the actual environment, the values are usually 1,2 , and $\infty$.

3.2. Genetic Algorithm. Many methods have been developed for traffic signal timing optimization. Conventional methods include integer programming and hill climbing. With the requirements of computing, intelligent algorithm is developed, such as genetic algorithm (GA). Using GA to solve

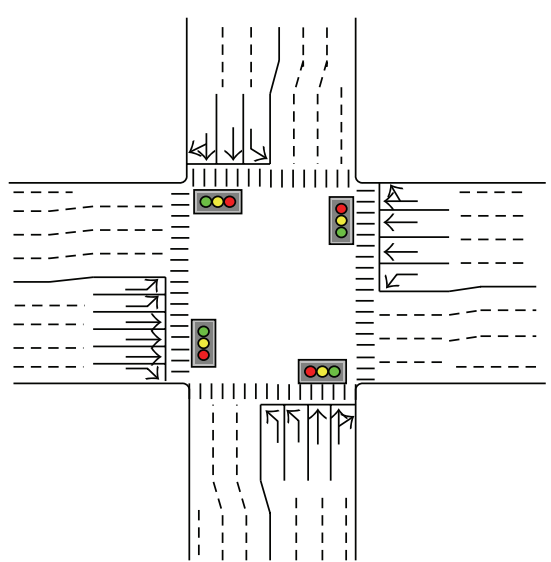

FIGURE 1: Geometry information of the intersection.

the fuzzy goal programming has been researched by $\mathrm{Pal}$ and Gupta [19]. The algorithm steps of solving the model are presented as follows.

Step 1 (representation and initialization). $M$ denotes the binary coded representation of a chromosome in a population as $M=\left\{g_{1}, g_{2}, \ldots, g_{n}\right\}$. Define pop-size as the population size and chromosomes are randomly initialized.

Step 2 (fitness function). Because the domain of Rosenbrock function is negative, the fitness function can be equal to the negative function. Namely, take the objective function $\left(Z^{\prime}=\right.$ $\left.-\min \left[\sum_{i=1}^{m}\left(\lambda_{i} u_{z i}\right)^{p}\right]^{1 / p}\right)$ as individual fitness in this paper.

Step 3 (take selection, crossover, and mutation operations). Classical roulette-wheel scheme is used for selecting in the genetic search process [5]. Choose proportional selection operator for selection operation, single point operator for crossover operation, and basic mutation operator for mutation operation.

\section{Model Verification}

A typical intersection in Changchun city is selected to verify the signal timing optimization method proposed in this paper. The intersection has four approaches and the geometrical characteristic is shown in Figure 1. There are four phases at this intersection. The first phase is used to release the straight traffic flow in the eastern and western approaches. The second phase is used to release the left traffic flow in the eastern and western approaches. Similarly, the third and the fourth are used to release the straight and left traffic flow in the southern and northern approaches, respectively.

The traffic flow of the intersection in different periods is investigated and the result is shown in Table 1. According to the investigation and the actual state of traffic flow at the intersection, set the saturation flow of left-turning traffic flow to $1700 \mathrm{pcu} / \mathrm{h}$. The saturation flow of straight flow and rightturning flow is set to $1800 \mathrm{pcu} / \mathrm{h}$ and $1550 \mathrm{pcu} / \mathrm{h}$, respectively. Besides, the saturation flow of straight and right mixed lane is set to $1700 \mathrm{pcu} / \mathrm{h}$. 
TABLE 1: Traffic flow in different periods ( $\mathrm{pcu} / \mathrm{h})$.

\begin{tabular}{lccccc}
\hline \multirow{2}{*}{ Turning direction } & \multicolumn{5}{c}{ Traffic volume } \\
& E & W & S & N & Y \\
\hline L & 201 & 249 & 386 & 272 & \\
T & 958 & 1130 & 850 & 915 & 0.8 \\
$\mathrm{R}$ & 261 & 240 & 76 & 103 & \\
\hline L & 187 & 204 & 238 & 204 & \\
$\mathrm{~T}$ & 764 & 810 & 508 & 583 & 0.6 \\
$\mathrm{R}$ & 190 & 150 & 52 & 82 & \\
\hline
\end{tabular}

TABLE 2: Extremum values of objective functions.

\begin{tabular}{lccccc}
\hline \multirow{2}{*}{ Traffic flow ratios } & \multicolumn{5}{c}{ Extremum values } \\
& Max/min & Cap & $D$ & $M_{\text {stop }}$ & $E_{j}$ \\
\hline \multirow{2}{*}{0.8} & Max & -2915 & 21803 & 263 & 31759 \\
& Min & -3267 & 7766 & 157 & 31537 \\
\hline \multirow{2}{*}{0.6} & Max & -2369 & 7682 & 98 & 31420 \\
& Min & -2872 & 2702 & 58 & 31341 \\
\hline
\end{tabular}

4.1. Signal Timing Parameters Optimization. The optimization objective functions can be established by using the actual traffic data above. Saturation flow and traffic volume are brought into formulas shown in Section 2.1 to obtain the four optimization objectives. Then a multiobjective function is built, of which the output variables are effective green times and signal cycle length. Constrains are explained in Section 2.2 in detail. To solve the models, genetic algorithm is employed through MATLAB software. The population size is 80 and iterations are 100 . The crossing rate and mutation rate are set as 0.8 and 0.05 . Through the above method, the minimum value and maximum value of each objective can be obtained which are shown in Table 2. When the traffic flow is high, the optimization goal is mainly focused on increasing traffic capacity and decreasing cycle vehicle delays. Therefore, the weight coefficients of these two objectives are bigger. It is known that the exhaust emission is mainly caused by the delay time. So the importance of exhaust emission is less than capacity and vehicle delay. Cycle vehicle stops are the last optimization objective in this state. When the traffic flow is low, the optimization goal is mainly focused on decreasing cycle vehicle delay and cycle vehicle stops. Then traffic capacity is optimized and finally exhaust emission is optimized.

Define the relationship value by using the evaluation function $v$ where $\alpha=0.1, \beta=0.9, \delta=0.3, \varepsilon=0.7$, and $\gamma=\nu=0.5$. As a consequence, preference relation matrixes $R_{1}$ and $R_{2}$ in two traffic flow states are established as follows:

$$
R_{1}=\left(\begin{array}{llll}
0.5 & 0.5 & 0.9 & 0.7 \\
0.5 & 0.5 & 0.9 & 0.7 \\
0.1 & 0.1 & 0.5 & 0.3 \\
0.3 & 0.3 & 0.7 & 0.5
\end{array}\right)
$$

$$
R_{2}=\left(\begin{array}{llll}
0.5 & 0.3 & 0.3 & 0.7 \\
0.7 & 0.5 & 0.5 & 0.9 \\
0.7 & 0.5 & 0.5 & 0.9 \\
0.3 & 0.1 & 0.1 & 0.5
\end{array}\right) .
$$

Calculate the weight coefficients of the membership functions and obtain $\lambda_{11}=\lambda_{12}=7 / 20, \lambda_{13}=1 / 12$, and $\lambda_{14}=13 / 60$ for the first case and $\lambda_{21}=13 / 60, \lambda_{22}=\lambda_{23}=$ $7 / 20$, and $\lambda_{24}=1 / 12$ for the second case.

Then transform the multiobjective function into single objective function for two states:

$$
\begin{aligned}
Z_{11} & =\max \left(\left(\frac{20405+7 Z_{1}}{-7040}\right)^{p}+\left(\frac{21803-7 Z_{2}}{280740}\right)^{p}\right. \\
& \left.+\left(\frac{263-Z_{3}}{1272}\right)^{p}+\left(\frac{412867-13 Z_{4}}{11460}\right)^{p}\right)^{1 / p}, \\
Z_{12} & =\max \left(\left(\frac{30797+13 Z_{1}}{-30180}\right)^{p}+\left(\frac{53774-7 Z_{2}}{99600}\right)^{p}\right. \\
& \left.+\left(\frac{686-7 Z_{3}}{800}\right)^{p}+\left(\frac{31420-Z_{4}}{948}\right)^{p}\right)^{1 / p} .
\end{aligned}
$$

For different values of $p$, the signal timing parameters are solved and optimized, including the cycle time and effective green times. The optimization processes by genetic algorithm through MATLAB software in different traffic states are illustrated in Figures 2 and 3. From Figures 2 and 3, it can be concluded that genetic algorithm can obtain the optimal solution quickly and the computation time is about 2 seconds which can meet the engineering requirements.

The signal timing scheme based on fuzzy compromise programming in this paper is denoted by Scheme 1 and the traditional scheme by Webster method is denoted by Scheme 2. Scheme 3 denotes the signal timing scheme obtained by adding the four objectives together simply and Scheme 4 denotes the signal timing scheme obtained by giving different weight values to diverse objectives according to traffic volume. In addition, three indexes are considered in Scheme 4 including intersection capacity, vehicle delays, and average vehicle stops. The classic weight coefficients according to traffic flow $[20,21]$ are adopted in Scheme 4 as follows:

$$
\begin{aligned}
k_{i}^{1} & =2 Y * \frac{C}{3600}, \\
k_{i}^{2} & =2 \sqrt[7]{s_{i}} *(1-Y), \\
k_{i}^{3} & =\sqrt[7]{s_{i}} * \frac{1-Y}{0.9} .
\end{aligned}
$$

The timing parameters optimization results obtained by different methods with high traffic flow and low traffic flow are shown in Tables 3 and 4, respectively. 

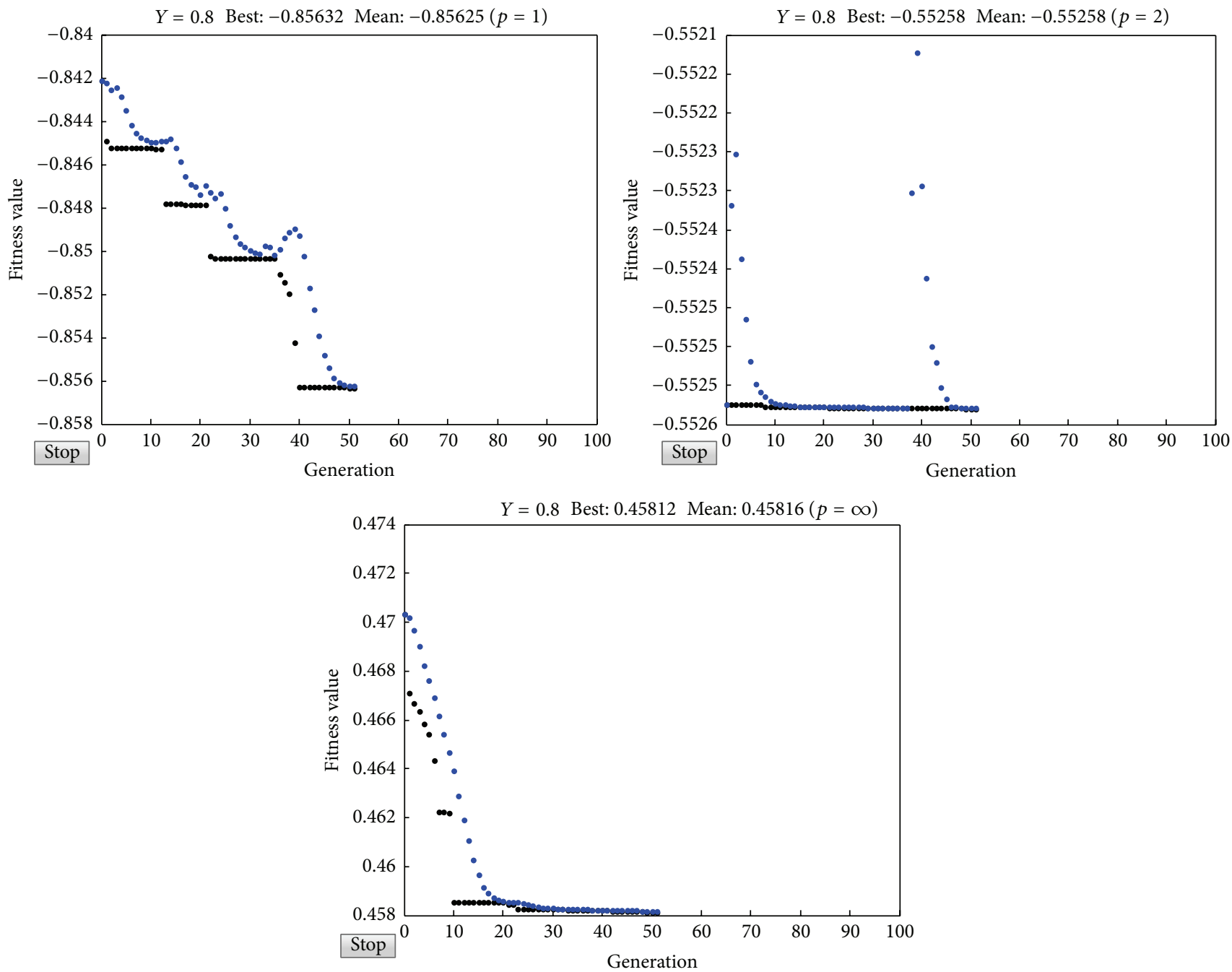

- Best fitness

- Mean fitness

FIGURE 2: Signal timing optimization process with high traffic flow.

TABLE 3: Signal timing optimization results with high traffic flow.

\begin{tabular}{|c|c|c|c|c|c|c|c|}
\hline \multirow{2}{*}{ Schemes } & \multicolumn{7}{|c|}{ Parameters } \\
\hline & $p$ & $Z$ & $g_{e 1}$ & $g_{e 2}$ & $g_{e 3}$ & $g_{e 4}$ & C \\
\hline \multirow{3}{*}{$\begin{array}{l}\text { Scheme } 1 \text { (fuzzy compromise } \\
\text { programming method) }\end{array}$} & 1 & 0.86 & 34 & 16 & 38 & 21 & 125 \\
\hline & 2 & 0.55 & 32 & 15 & 37 & 20 & 120 \\
\hline & $\infty$ & 0.46 & 33 & 16 & 37 & 21 & 123 \\
\hline Scheme 2 (Webster method) & - & - & 37 & 19 & 47 & 26 & 145 \\
\hline Scheme 3 (simply weighted) & - & - & 32 & 18 & 38 & 21 & 125 \\
\hline Scheme 4 (weighted by volume) & - & - & 36 & 18 & 37 & 23 & 130 \\
\hline
\end{tabular}

4.2. Simulation Results Analysis. The operation performance of the signal timing schemes is compared and analyzed in VISSIM simulation software. Because the exhaust emission has high consistency with vehicle delays in the performance, so the vehicle delays, vehicle average stops, vehicle throughput numbers, and total stops are selected as the evaluation indexes. The simulation time is set to $7200 \mathrm{~s}$ and the time interval is set to $600 \mathrm{~s}$. The traffic running effects in different flow ratios and schemes are illustrated in Figures 4 and 5, respectively.

Through comparison and analysis, it can be concluded that the optimized scheme in this paper can effectively 

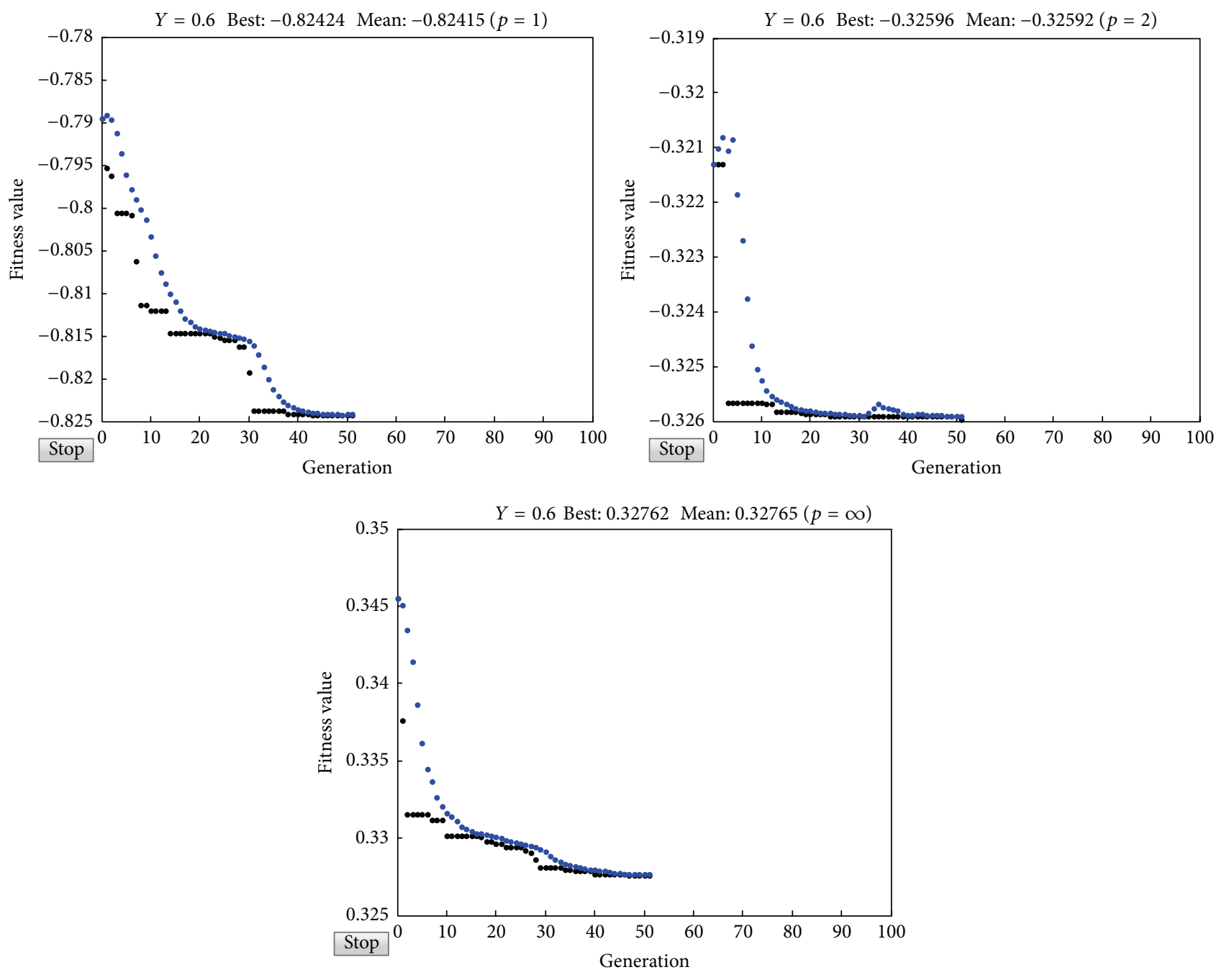

- Best fitness

- Mean fitness

FIGURE 3: Signal timing optimization process with low traffic flow.

TABLE 4: Signal timing optimization results with low traffic flow.

\begin{tabular}{|c|c|c|c|c|c|c|c|}
\hline \multirow{2}{*}{ Schemes } & \multicolumn{7}{|c|}{ Parameters } \\
\hline & $p$ & $Z$ & $g_{e 1}$ & $g_{e 2}$ & $g_{e 3}$ & $g_{e 4}$ & C \\
\hline \multirow{3}{*}{ Scheme 1 (fuzzy compromise programming method) } & 1 & 0.82 & 15 & 10 & 13 & 10 & 64 \\
\hline & 2 & 0.33 & 14 & 10 & 14 & 10 & 64 \\
\hline & $\infty$ & 0.33 & 14 & 10 & 14 & 11 & 66 \\
\hline Scheme 2 (Webster method) & - & - & 17 & 10 & 18 & 11 & 72 \\
\hline Scheme 3 (simply weighted) & - & - & 17 & 10 & 15 & 10 & 68 \\
\hline Scheme 4 (weighted by volume) & - & - & 17 & 10 & 16 & 11 & 70 \\
\hline
\end{tabular}

reduce total vehicle delays and improve vehicle throughput numbers in a time interval and the optimization effect is obvious whether the traffic flow ratio is high or low. However, the improvement of vehicle stops is not obvious as the other two performance indexes. From Figure 4, Scheme 1, Scheme 3, and Scheme 4 can reduce vehicle delays effectively when traffic flow is high, compared with Scheme 2
(Webster method). It illustrates that multiobjective optimization method is more effective than traditional single objective optimization method. The vehicle delays of Scheme 4 are more than Scheme 3 and less than Scheme 2, which proves that four-objective optimization is better than three-objective optimization. Besides, the method proposed in this paper denoted by Scheme 1 performs best in the four schemes. 

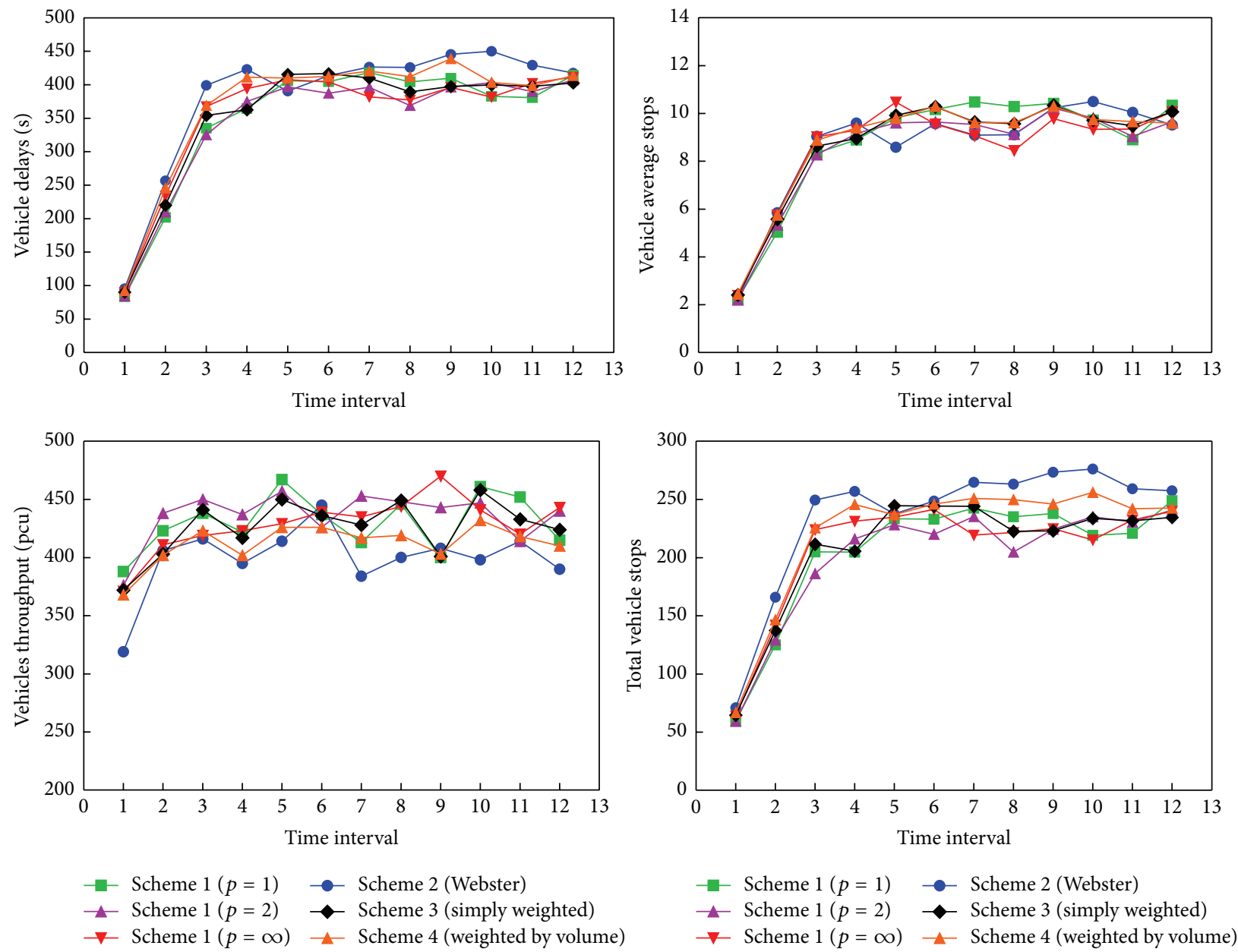

FIgURE 4: Performance of different schemes with high traffic flow.

The reason is that Scheme 1 considers the whole running benefits of the intersection and avoids the subjectivity of the weight coefficients. Although the optimization effect of vehicle average stops is not obvious, the throughput vehicles and total vehicle stops are optimized to be the best of the proposed method in this paper. From Figure 5, when traffic flow is low, Scheme 1 can reduce vehicle delays and total vehicle stops effectively, compared with other schemes. The vehicle average stops and throughput vehicles optimization effects are not obvious. The reason may be that traffic demand is not high and traffic flow runs smoothly. Therefore, the proposed method in this paper is efficient and performs best.

Tables 5 and 6 illustrate the comparisons of average operation efficiency for different traffic flow ratios, respectively. Compared with Scheme 2 obtained by Webster method, Scheme 1 presented in this paper optimizes the average total delays obviously under different values of $p$. When the traffic flow is high, the improvement ratios of average total delays are $8.57 \%, 9.8 \%$, and $7.28 \%$ for $p=1, p=2$, and $p=$ $\infty$, respectively. Similarly, when the traffic flow is low, the improvement ratios are $21.3 \%, 20.5 \%$, and $17.7 \%$. It is obvious that the optimization effect is more significant in low traffic flow state than high traffic flow. The optimization of vehicle average stops is not obvious as the delays. For high traffic flow, the improvement ratios are $0 \%, 2.1 \%$, and $1 \%$. And for low traffic flow, the improvement ratios are bigger which are $7.6 \%$, $8.4 \%$, and $6.7 \%$. However, the optimization of total vehicle stops is obvious and the improvement ratios are $13.15 \%$, $14.91 \%$, and $11.97 \%$ for high traffic flow and $16.10 \%, 15.25 \%$, and $11.02 \%$ for low traffic flow. The improvements are very obvious. Results also show that vehicle throughput number in a time interval is optimized and the improvement ratios are $7.8 \%, 9.2 \%$, and $7.4 \%$ for high traffic flow and $1.1 \%, 1 \%$, and $0 \%$ for low traffic flow. As a consequence of the analysis above, the optimized signal timing scheme of this paper is better than Webster scheme, which is proved to improve the overall efficiency of the intersection to a certain extent significantly. In particular, the optimization performance is best when $p=$ 2.

Compared with Scheme 3 obtained by adding the four objectives together simply, Scheme 1 can optimize the four performance indexes. The improvement ratios of average total delays are $1.62 \%, 2.93 \%$, and $0.22 \%$ for high traffic flow and $12.39 \%, 11.55 \%$, and $8.45 \%$ for low traffic flow. For high traffic flow, the improvement ratios of average stops are $0.34 \%, 2.95 \%$, and $1.93 \%$. And for low traffic flow, 

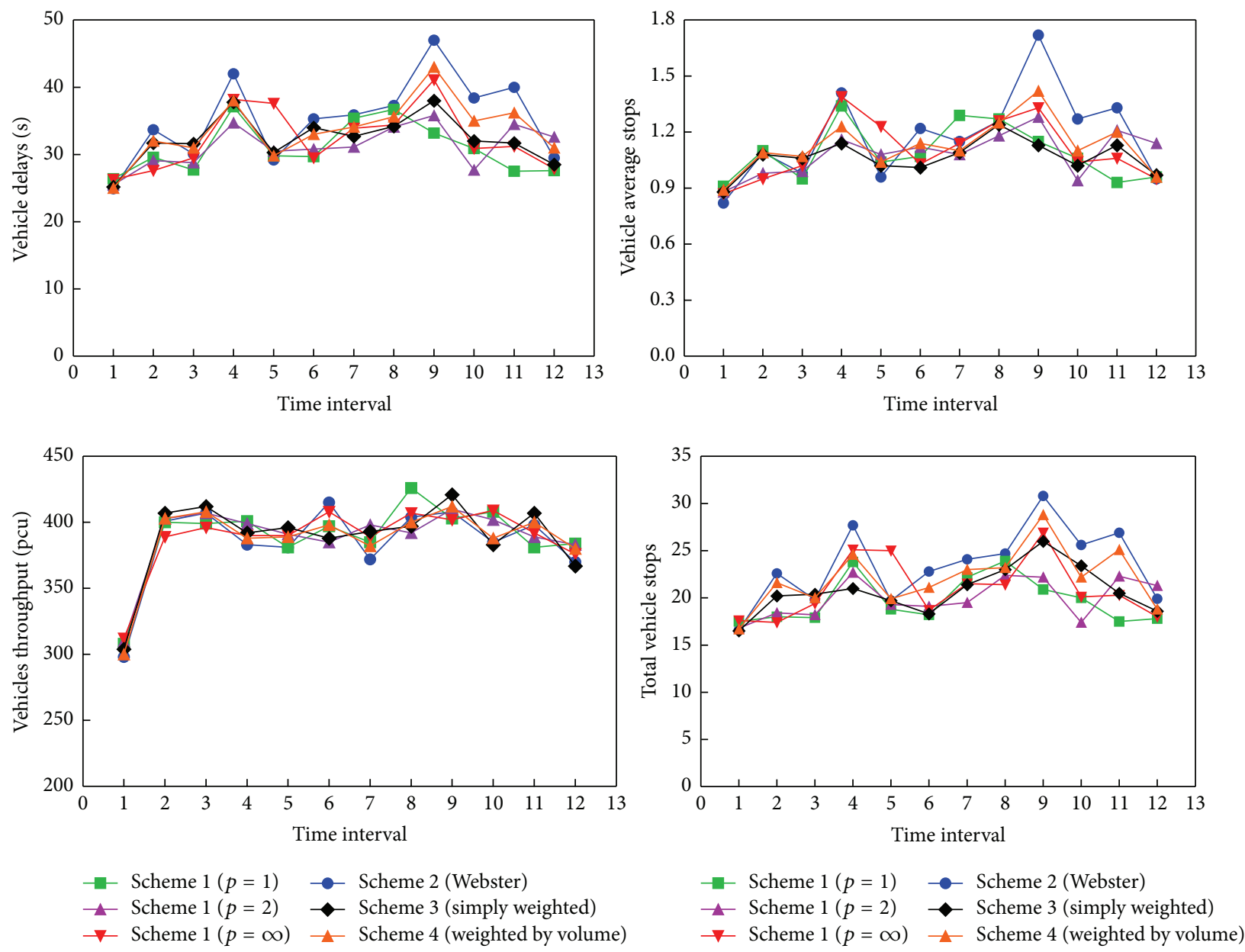

Figure 5: Performance of different schemes with low traffic flow.

TABLE 5: Average performance comparison with high traffic flow.

\begin{tabular}{lccccc}
\hline \multirow{2}{*}{ Schemes } & & \multicolumn{3}{c}{ Parameters } \\
& $p$ & Delay & Average stop & Vehicles throughput & Total stops \\
\hline & 1 & 353 & 8.78 & 5162 & 206.8 \\
Scheme 1 (fuzzy compromise programming method) & 2 & 348.3 & 8.55 & 5229 & 202.6 \\
& $\infty$ & 358 & 8.64 & 8145 & 4789 \\
Scheme 2 (Webster method) & - & 386.1 & 8.73 & 5112 & 238.1 \\
Scheme 3 (simply weighted) & - & 358.8 & 8.81 & 5005 & 210.5 \\
Scheme 4 (weighted by volume) & & 368.1 & 8.98 & 217 \\
\hline
\end{tabular}

TABLE 6: Average performance comparison with low traffic flow.

\begin{tabular}{|c|c|c|c|c|c|}
\hline \multirow{2}{*}{ Schemes } & \multicolumn{5}{|c|}{ Parameters } \\
\hline & $p$ & Delay & Average stop & Vehicles throughput & Total stops \\
\hline \multirow{3}{*}{ Scheme 1 (fuzzy compromise programming method) } & 1 & 31.1 & 1.1 & 4673 & 19.8 \\
\hline & 2 & 31.4 & 1.09 & 4669 & 20 \\
\hline & $\infty$ & 32.5 & 1.11 & 4660 & 21 \\
\hline Scheme 2 (Webster method) & - & 39.5 & 1.19 & 4622 & 23.6 \\
\hline Scheme 3 (simply weighted) & - & 35.5 & 1.13 & 4667 & 22.1 \\
\hline Scheme 4 (weighted by volume) & & 35.6 & 1.19 & 4634 & 23.4 \\
\hline
\end{tabular}


the improvement is more obvious and the ratios are $2.66 \%$, $3.54 \%$, and $1.77 \%$. Results also show that vehicle throughput number is optimized and improvement ratios are $0.98 \%$, $2.29 \%$, and $0.65 \%$ for high traffic flow and $0.13 \%, 0.04 \%$, and $0.15 \%$ for low traffic flow. Total stops improvement ratios are $1.76 \%, 3.75 \%$, and $0.43 \%$ for high traffic flow and $10.41 \%, 9.50 \%$, and $4.98 \%$ for low traffic flow. In summary, the improvement is less than Scheme 2 which proves that the scheme considering multiple objectives performs better.

Compared with Scheme 4 obtained by considering three indexes and weighted by volume, Scheme 1 performs better. Average total delays improvements are $4.10 \%, 5.38 \%$, and $2.74 \%$ for high traffic flow and $12.64 \%, 11.80 \%$, and $8.71 \%$ for low traffic flow. The improvement ratios of average stops are $2.23 \%, 4.79 \%$, and $3.79 \%$ for high traffic flow and $7.56 \%$, $8.40 \%$, and $6.72 \%$ for low traffic flow. For high traffic flow, the improvement of vehicle throughput number is obvious and ratios are $3.14 \%, 4.48 \%$, and $2.80 \%$. For low traffic flow, the improvements are $0.84 \%, 0.76 \%$, and $0.56 \%$. Total stops improvement ratios in an interval are $4.7 \%, 6.64 \%$, and $3.41 \%$ for high traffic flow and $15.39 \%, 14.53 \%$, and $10.26 \%$ for low traffic flow which is more obvious.

To sum up, Schemes 1, 3, and 4 all perform better than Scheme 2 which proves that multiobjective signal optimization method is more effective than single objective signal optimization method. Scheme 1 performs better than Scheme 3 and Scheme 4. Besides, Scheme 3 performs better than Scheme 4. Therefore, Scheme 1 performs the best. In particular, the optimization performance is the best when $p=2$. All the analysis above proves that the method proposed in this paper is effective and better.

\section{Conclusions}

The signal timing optimization of isolated intersection is researched in this paper. Considering the overall running efficiency of the intersection comprehensively, a multiobjective function is established first of which the optimization objectives include traffic capacity, cycle vehicle delays, cycle stops, and exhaust emission at the intersection. Then fuzzy compromise programming method is employed to convert the multiobjective function to a single objective function by giving different objectives diverse weight coefficients according to traffic flow ratios. To solve the models, genetic algorithm is applied by programming in MATLAB software. As a consequence, the optimized signal cycle and effective green time are obtained. Finally, the optimized signal timing presented in this paper is verified by an actual intersection in Changchun city. Input the related investigation data and build simulation environment through VISSIM software. Simulation results show that the signal timing based on fuzzy compromise programming in this paper can significantly improve traffic capacity and effectively reduce vehicle delays and stops, compared with traditional methods. To sum up, the method proposed in this paper is effective and correct.

In this paper, signal timing optimization of unsaturated isolated intersection is researched and saturated and oversaturated intersections are not considered. Because of the limitations, it is advisable to take the signal timing optimization in saturated state and oversaturated state into account with more field data collection efforts in the future research.

\section{Competing Interests}

The authors declare that they have no competing interests.

\section{Acknowledgments}

This research has been jointly supported by National High Technology Research and Development Plan Project (Grant no. 2014BAG03B03) and National Natural Science Foundation of China (Grants nos. 51308248 and 51408257).

\section{References}

[1] F. V. Webster and B. M. Cobbe, Traffic Signals, Ministry of Transport, Her Majesty's Stationery Office, London, UK, 1966.

[2] A. Ceder and I. Reshetnik, "An algorithm to minimize queues at signalized intersections," Journal of the Operational Research Society, vol. 52, no. 6, pp. 615-622, 2001.

[3] P. Wang and Q. Yang, "Genetic algorithms based traffic signal optimization at a congested intersection," Applied Mechanics and Materials, vol. 209-211, pp. 814-817, 2012.

[4] H. C. Liu, K. N. Balke, and W.-H. Lin, "A reverse causal-effect modeling approach for signal control of an oversaturated intersection," Transportation Research Part C: Emerging Technologies, vol. 16, no. 6, pp. 742-754, 2008.

[5] W. Deng, L. M. Jia, and H. H. Dong, "Multi-objective optimization model for urban traffic intersection control based on data fusion," Advanced Materials Research, vol. 156-157, pp. 505-510, 2011.

[6] P. Jiao and T. Sun, "Multiobjective traffic signal control model for intersection based on dynamic turning movements estimation," Mathematical Problems in Engineering, vol. 2014, Article ID 608194, 8 pages, 2014.

[7] J. Anderson, T. Sayers, and M. Bell, "The objectives of traffic signal control," Journal of Traffic Engineering \& Control, vol. 3, pp. 167-170, 1998.

[8] J.-D. Schmöcker, S. Ahuja, and M. G. H. Bell, "Multi-objective signal control of urban junctions-framework and a London case study," Transportation Research Part C: Emerging Technologies, vol. 16, no. 4, pp. 454-470, 2008.

[9] Y. Li, L. J. Yu, S. R. Tao, and K. M. Chen, "Multi-objective optimization of traffic signal timing for oversaturated intersection," Mathematical Problems in Engineering, vol. 2013, Article ID 182643, 9 pages, 2013.

[10] Y. S. Murat and E. Gedizlioglu, "A fuzzy logic multi-phased signal control model for isolated junctions," Transportation Research Part C: Emerging Technologies, vol. 13, no. 1, pp. 19-36, 2005.

[11] Transportation Research Board, Highway Capacity Manual 2000, National Research Council, Federal Highway Administration, Washington, DC, USA, 2000.

[12] Y. S. Quan, Urban Traffic Control, Renmin Jiaotong Press, Beijing, China, 1989.

[13] R. Akçelik and N. M. Rouphail, "Estimation of delays at traffic signals for variable demand conditions," Transportation Research Part B, vol. 27, no. 2, pp. 109-131, 1993. 
[14] X. Li, G. Li, S.-S. Pang, X. Yang, and J. Tian, "Signal timing of intersections using integrated optimization of traffic quality, emissions and fuel consumption: a note," Transportation Research Part D: Transport and Environment, vol. 9, no. 5, pp. 401-407, 2004.

[15] M. Barth, "Vehicle emissions modeling in the U.S.A," in Proceedings of the 3rd Japan Clean Air Program Conference, pp. 6772, Tokyo, Japan, 2002.

[16] A. K. Bit, M. P. Biswal, and S. S. Alam, "Fuzzy programming approach to multiobjective solid transportation problem," Fuzzy Sets and Systems, vol. 57, no. 2, pp. 183-194, 1993.

[17] L. Li and K. K. Lai, "A fuzzy approach to the multiobjective transportation problem," Computers \& Operations Research, vol. 27, no. 1, pp. 43-57, 2000.

[18] N. Cetin and F. Tiryaki, "A fuzzy approach using generalized dinkelbach's algorithm for multiobjective linear fractional transportation problem," Mathematical Problems in Engineering, vol. 2014, Article ID 702319, 10 pages, 2014.

[19] B. B. Pal and S. Gupta, "A genetic algorithm approach to fuzzy goal programming formulation of fractional multiobjective decision making problems," in Proceedings of the 1st International Conference on Advanced Computing (ICAC '09), pp. 5560, IEEE, Chennai, India, December 2009.

[20] H. Z. Gu and W. Wang, "A global optimization simulated annealing algorithm for intersection signal timing," Journal of Southeast University, vol. 28, no. 3, pp. 68-72, 1998.

[21] X.-H. Chen, D.-L. Qian, and D.-H. Shi, "Multi-objective optimization method of signal timing for the non-motorized transport at intersection," Journal of Transportation Systems Engineering and Information Technology, vol. 11, no. 2, pp. 106111, 2011. 


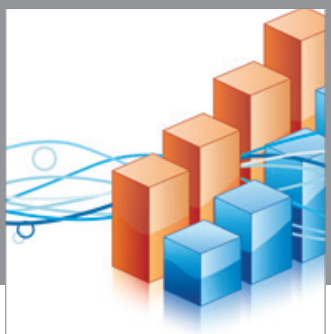

Advances in

Operations Research

vatem alat4

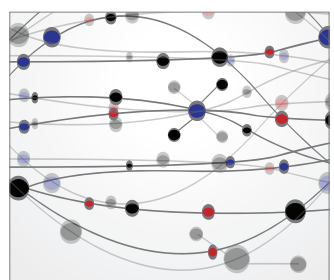

\section{The Scientific} World Journal
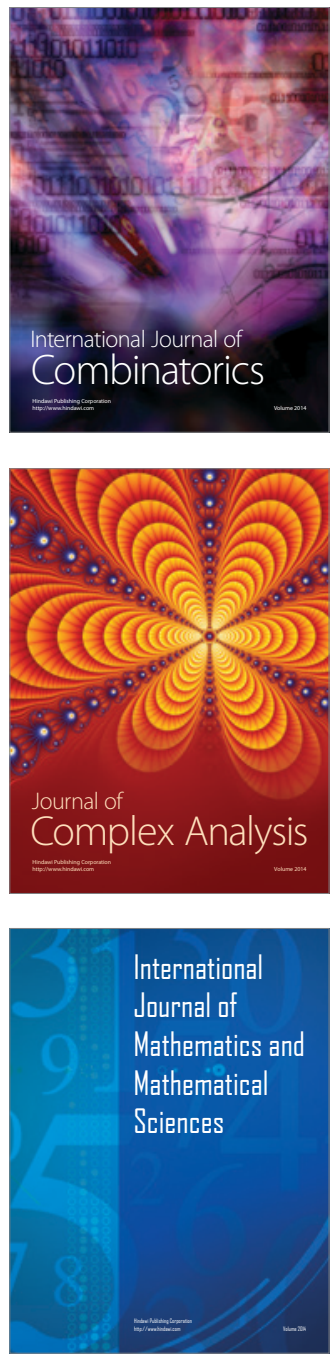
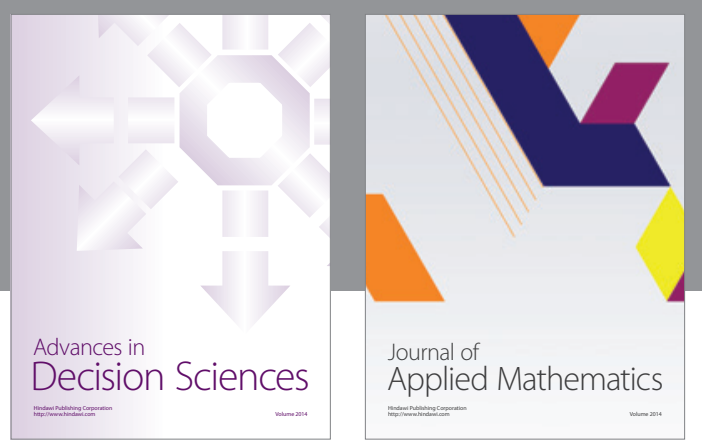

Algebra

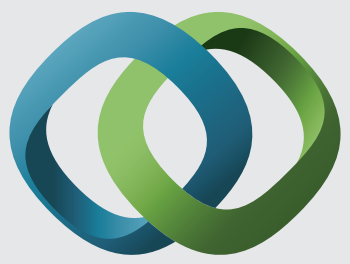

\section{Hindawi}

Submit your manuscripts at

http://www.hindawi.com
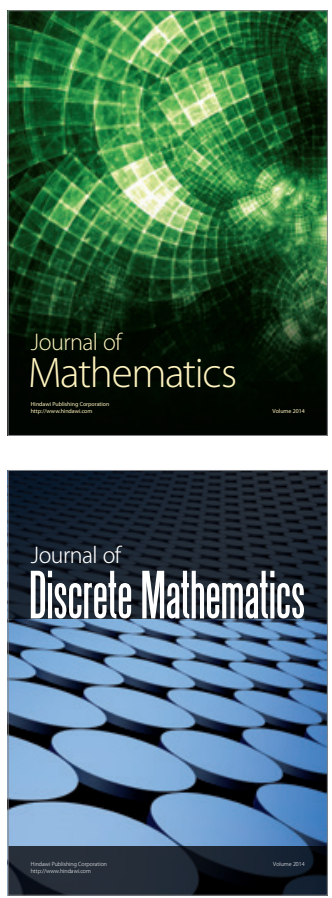

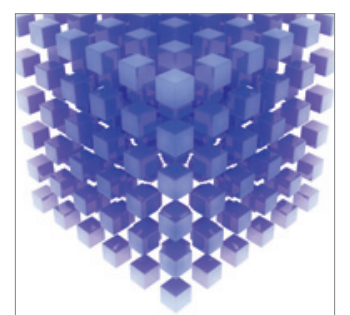

Mathematical Problems in Engineering
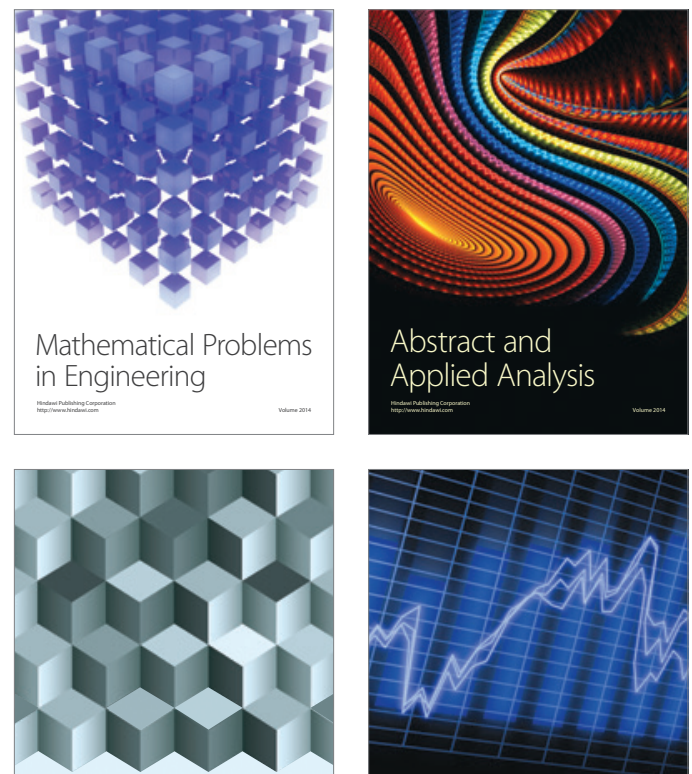

Journal of

Function Spaces

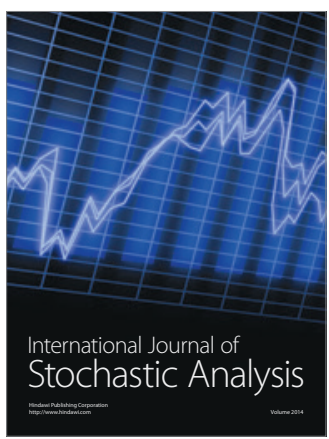

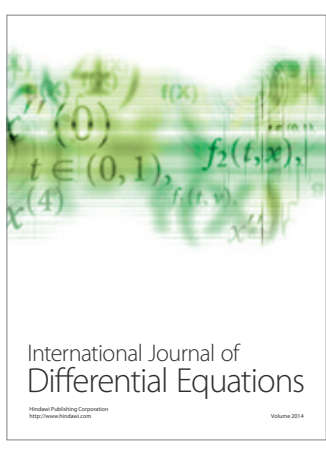
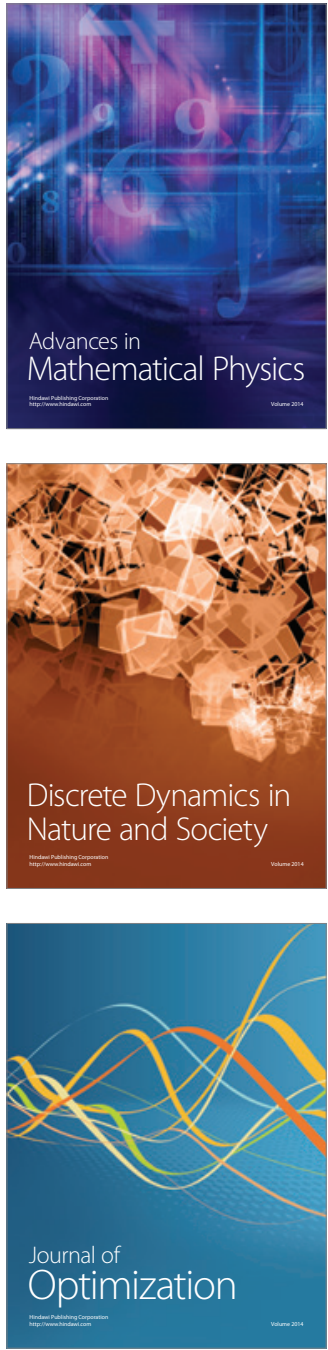